\title{
A REVIEW ON CONTENT BASED IMAGE RETRIEVAL
}

\author{
Nitika Seth (1), Sonika Jindal (2) \\ (1) Research Scholar, Department of Computer Science Engineering, SBSSTC, Ferozepur \\ nitikaseth15@gmail.com \\ (2) Assistant Professor, Department of Computer Science Engineering, SBSSTC, Ferozepur \\ sonikamanoj@gmail.com
}

\begin{abstract}
Image retrieval means to recover the original image from the reconstructed image, here in this paper we have discussed latest techniques in the field of image retrieval for image processing. Content Based Image Retrieval (CBIR) is one of the most exciting and fastest growing research areas in the field of Image Processing. The techniques presented are Boosting image retrieval, soft query in image retrieval system, content based image retrieval by integration of metadata encoded multimedia features, and object based image retrieval and Bayesian image retrieval system. Some probable future research directions are also presented here to explore research area in the field of image retrieval
\end{abstract}

\section{Keywords}

Image processing, Local binary pattern, cbir, opencv.

\section{INTRODUCTION}

Content-based image retrieval (CBIR) systems demonstrate excellent performance at computing low-level features from pixel representations but its output does not reflect the overall desire of the user. The systems perform poorly in extracting high-level (semantic) features that include objects and their meanings, actions and feelings. This phenomenon, referred to as the semantic gap, has necessitated current research in CBIR systems towards retrieving images by the type of object or scene depicted. Analyzing and interpretation of image data in large and diverse image database, as in a CBIR system is obviously difficult because there is no prior information on the size or scale of individual structures within the images to be analysed. Content-Based Image Retrieval, commonly referred to as CBIR, is the automatic retrieval of digital images from large databases. This technique makes use of the inherent visual contents of an image to perform a query. As opposed to earlier image retrieval methods which involved the manual textual annotations of images, CBIR systems identify the images by automaticallyextracted syntactical features. With the advance in technology, including the everincreasing popularity of digital cameras and the possibility to manage and store large databases of information, CBIR proves to be much more efficient and practical. It relieves the user from the previous cumbersome, subjective and errorprone task of image description and has therefore dramatically improved the usability of the system.

\section{THE USE OF IMAGES}

Historical records show the use of images date back to paintings on walls of cave by early man. In the pre-Roman times images were seen mostly in the form of building plans and maps [1]. The need and use of images grew with the ages, particularly with the advent of photography in the sixteenth century. In the twentieth century, introduction of computer and advances in science and technology gave birth to low cost and efficient digital storage devices and the worldwide web, which in turn became the catalyst for increasing acquisition of digital information in the form of images.

In this computer age virtually all spheres of human life including commerce, government, academics, hospitals, crime prevention, surveillance, engineering, architecture, journalism, fashion and graphic design and historical research are in need of, and use of images for efficient services. A large collection of images is referred to as image database. Image database is a system where image data are integratedly stored [2]. Image data include the raw images and information extracted from images by automated or computer assisted image analysis.

The police maintain image database of criminals, crime scenes and stolen items. In the medical profession X-rays, and scanned image database are kept for diagnosis, monitoring and research purposes. In architectural and engineering design image database exist for design projects, finished projects and machine parts. In publishing and advertising journalists create image database for various events and activities such as sports, buildings, personalities, national and international events, and product advertisements. In historical research image database are created for archives in areas that include arts, sociology and medicine.

\section{IMAGE RETRIEVAL PROBLEM}

In small collection of images simple browsing can identify an image. This is not the case for large and varied collection of images, where the user encounters image retrieval problem. Image retrieval problem is the problem of searching and retrieving images that are relevant to a user's request from a database. A typical retrieval problem example is a design engineer who needs to search his organisation database for design projects similar to that required by his clients or the police seeking to confirm the face of a suspected criminal among faces in the database of renowned criminals. In the commerce department before trademark is finally approved for use there is need to find out if such or similar ones ever existed. In the hospital some ailments require the medical practitioner to search and review similar X-rays or scanned images of a patient before proffering solution.

\section{VISUAL CONTENT LEVELS}


Images are naturally endowed with attributes or information content that can help in resolving the image retrieval problem. The information content that can be derived from an image is classified into three levels. See Figure.1

- Low level - They include visual features such as colour, texture, shape, spatial information and motion.

- Middle level - Examples include presence or arrangement of specific types of objects, roles and scenes.

- High level - Include impressions, emotions and meaning associated with the combination of perceptual features. Examples include objects or scenes with emotional or religious significance.
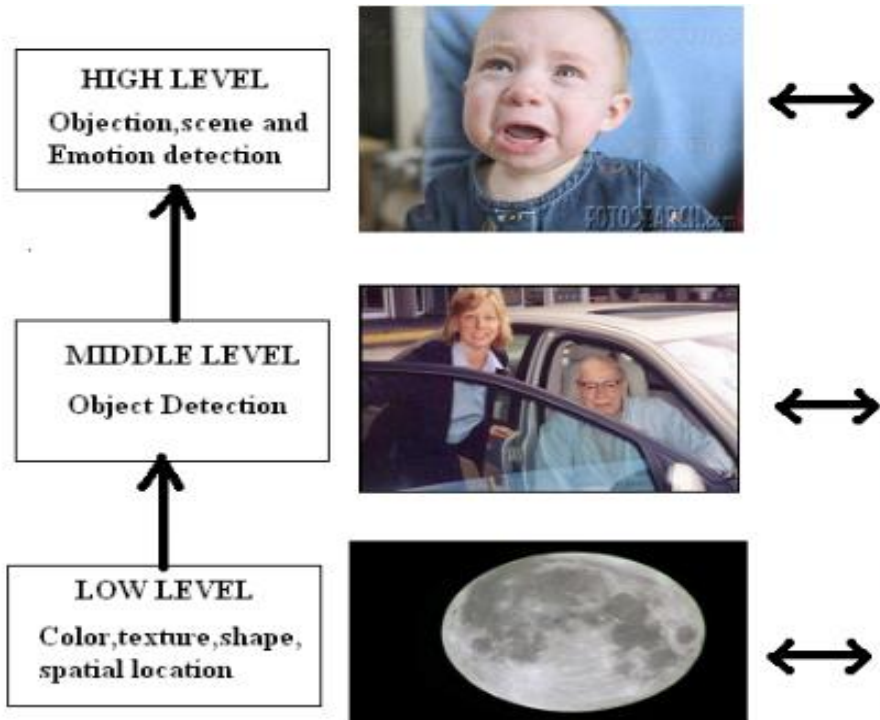

CRYING
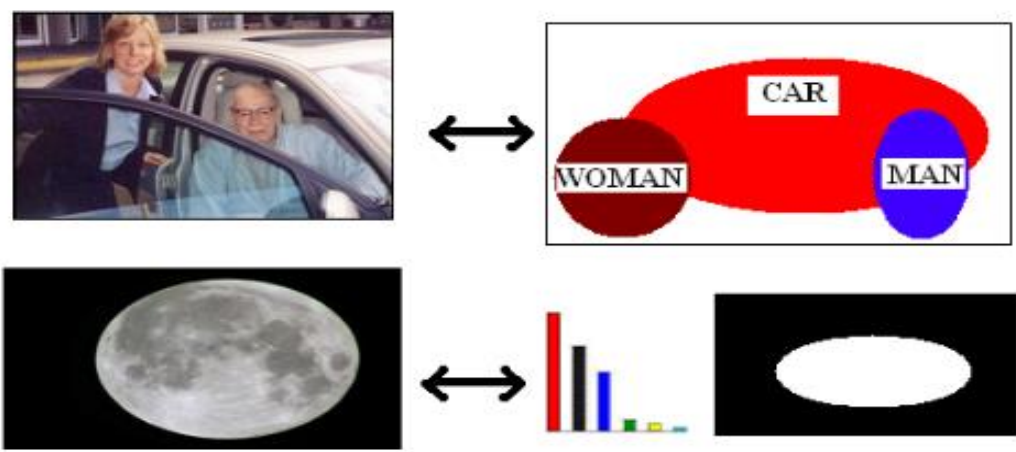

Figure 1. Image Content Levels

The image content level is also a measure of level of feature extraction. At the low level, also regarded as primary level the features extracted (color, shape, texture,spatial information and motion) are called primitive features because they can only be extracted by information obtained at the pixel level, that is pixel representation of the images. The middle level features are features that can be extracted by collection of pixels that make up the image, while high level features goes beyond the collection of pixels. It identifies the impressions, meanings and emotions associated with the collection of pixels that make up the object.

\section{TEXT-BASED RETRIEVAL AND CONTENT-BASED RETRIEVAL}

An image retrieval system is a computer system for browsing, searching and retrieving images in an image database. Text-based and content-based are the two techniques adopted for search and retrieval in image database.

In text-based retrieval, images are indexed using keywords, subject headings or classification codes, which in turn are used as retrieval keys during search and retrieval.Text-based retrieval is non-standardized because different users use different keywords for annotation. Text descriptions are sometimes subjective and incomplete because it cannot depict complicated image features very well. Examples are texture images that cannot be described by text. In text retrieval, humans are required to personally describe every image in the database, so for a large image database the technique is cumbersome, expensive and labour-intensive.

Content-based image retrieval (CBIR) technique use image content to search and retrieve digital images . Content-based image retrieval system was introduced to address the problems associated with text-based image retrieval, [3]. Advantages of content-based image retrieval over text-based retrieval will be mentioned in the next sections.

However, text-based and content-based image retrieval techniques complement each other. Text-based techniques can capture high-level feature representation and concepts. It is easy to issue text queries but text-based techniques cannot accept pictorial queries. On the other hand, content-based techniques can capture low-level image features and accept pictorial queries. But they cannot capture high-level concepts effectively. Retrieval systems exist which combine both techniques for more efficient retrieval.

\section{PRINCIPLE OF CBIR}

A typical CBIR system as shown in Figure.2 automatically extract visual attributes (colour, shape, texture and spatial information) of each image in the database based on its pixel values and stores in a different database within the system called feature database. The feature data for each of the visual attributes of each image is very much smaller in size compared to the image data. Thus the feature database contains an abstraction (compact form) of the images in the 
image database; each image is represented by a compact representation of its contents (colour, texture, shape and spatial information) in the form of a fixed length real-valued multi-component feature vectors or signature.

The users usually formulate query image and present to the system. The system automatically extract the visual attributes of the query image in the same mode as it does for each database image, and then identifies images in the database whose feature vectors match those of the query image, and sorts the best similar objects according to their similarity value.

During operation the system processes less compact feature vectors rather than the large size image data thus giving CBIR its cheap, fast and efficient advantage over text-based retrieval.

CBIR system can be used in one of two ways. First, exact image matching, that is matching two images, one an example image and the other, image in image database. Second is approximate image matching, which is finding most closely match images to a query image.

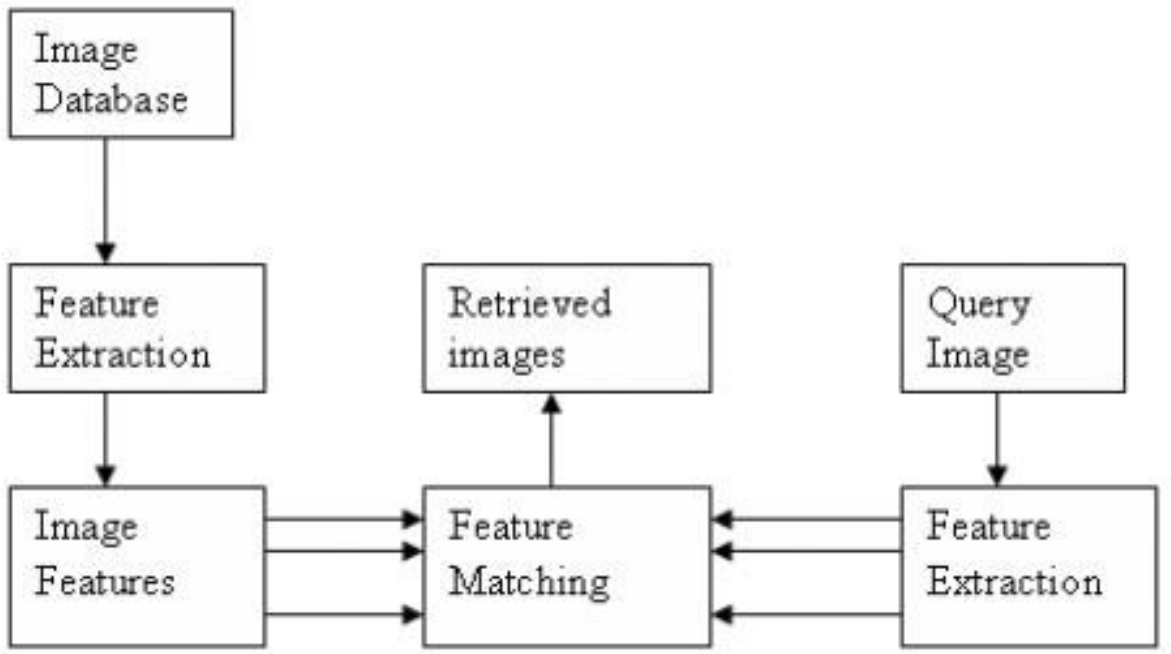

Figure 2. Process Involved in Content - based image retrieval

\section{RELATED WORK}

James Z. Wang et al. (2001) presented simplicity (Semantics sensitive Integrated Matching for Picture Libraries), an image retrieval system, which uses semantics classification methods, a wavelet-based approach for feature extraction, and integrated region matching based upon image segmentation. As in other region based retrieval systems, an image is represented by a set of regions, roughly corresponding to objects, which are characterized by color, texture, shape, and location.

Yixin Chen et al. (2002) proposes a fuzzy logic approach, UFM (unified feature matching), for region-based image retrieval. In their retrieval system, an image is represented by a set of segmented regions, each of which is characterized by a fuzzy feature (fuzzy set) reflecting color, texture, and shape properties. As a result, an image is associated with a family of fuzzy features corresponding to regions.

Yixin Chen et al. (2005) introduces a new technique, cluster-based retrieval of images by unsupervised learning (CLUE), for improving user interaction with image retrieval systems by fully exploiting the similarity information. CLUE retrieves image clusters by applying a graph-theoretic clustering algorithm to a collection of images in the vicinity of the query. Clustering in CLUE is dynamic.

R. Fergus et al. (2005) developed a new model, TSI-pLSA, which extends pLSA (as applied to visual words) to include spatial information in a translation and scale invariant manner. Their approach can handle the high intra-class variability and large proportion of unrelated images returned by search engines.

Savvas A. Chatzichristofis et al. (2008) deals with a new low level feature that is extracted from the images and can be used for indexing and retrieval. This feature is called "Color and Edge Directivity Descriptor" and incorporates color and texture in formation in a histogram.

Chuen-Horng Lin et al. (2008) proposes three feature vectors for image retrieval. In addition, a feature selection technique is also brought forward to select optimal features to not only maximize the detection rate but also simplify the computation of image retrieval. The first and second image features are based on color and texture features, respectively called color co-occurrence matrix (CCM) and difference between pixels of scan pattern (DBPSP) in this research work.

Michal Perdoch et al. (2009) proposes a novel method for learning discretized local geometry representation based on minimization of average reprojection error in the space of ellipses. The representation requires only 24 bits per feature without drop in performance. 
Herve Jegou et al. (2010) addresses the problem of image search on a very large scale, where three constraints have to be considered jointly the accuracy of the search, its efficiency, and the memory usage of the representation.

Yanzhi Chen et al. (2012) proposed a discriminative criterion for improving result quality. This criterion lends itself to the addition of extra query data, and they showed that multiple query images can be comb index to produce enhanced results. Experiments compare the performance of the method to state-of-the-art in object retrieval, and show how performance is lifted by the inclusion of further query images.

Relja Arandjelovi'c et al. (2012) made the following three contributions: (i) a new method to compare SIFT descriptors (RootSIFT) which yields superior performance without increasing processing or storage requirements; (ii) a novel method for query expansion where a richer model for the query is learnt discriminatively in a form suited to immediate retrieval through efficient use of the inverted index; (iii) an improvement of the image augmentation method proposed by Turcot and Lowe where only the augmenting features which are spatially consistent with the augmented image are kept.

Sumaira Muhammad et al. (2012) has given comparison of three different approaches of CBIR based on image features and similarity measures taken for finding the similarity between two images. Results have shown that selecting an important image feature and calculating that through a meaningful way is of great importance in image retrieval.

Sreedevi S et al. (2013) proposed a fast image retrieval algorithm called feature levels. Feature levels algorithm works with the classification of image features to different categories or levels, feature extraction in terms of levels and feature similarity comparison of the query image with database images.

Soundararajan Ezekiel et al. (2013) explored contourlet transformation in association with Pulse Coupled Neural Network (PCNN) while the second technique is based on Rescaled Range (R/S) Analysis. Both methods provide flexible multiresolution decomposition, directional feature extraction and are suitable for image fusion.

Hui Xie et al. (2013) puts forward an analogy-relevance feedback (analogy-RF) CBIR method using multiple features which only needs one. The method allows users to choose the kind of object of the query image when they input the query image, and their system can determine several analogy-RF images in the sample database.

Khadidja et al. (2013) mainly reviews and compares different approaches of CBIR using RF. Its ultimate goal is to present information about image database aspects and image features setting so as to support the selection of the appropriate CBIR with RF Techniques.

Sandeep Kumar et al. (2014) proposed a parallel approach to the morphological feature extraction process and demonstrated a good computational speedup. Remote sensing images have a typical property of incrementing constantly and each image being very large.

Komal Juneja et al. (2015) presented a survey on low-level feature description techniques for Content Based Image Retrieval is presented with its various applications. In the modern era, with the explosive growth of image databases, huge amount of image and video archive led to rise of a new research and development of efficient method to searching, locating and retrieving of image.

Ghanshyam Raghuwanshi et al. (2015) proposes a novel technique for texture image retrieval based on tetrolet transforms. Tetrolets provide fine texture information due to its different way of analysis. Tetrominoes are applied at each decomposition level of an image and best combination of tetrominoes is selected, which better shows the geometry of an image at each level.

Savita Gandhani et al. (2013) presented a novel approach in content-based image retrieval (CBIR) by combining the lowlevel feature i.e. color, texture and shape features. At first, they are transforming the color space from RGB model to HSV model, and then extracting color histogram to form color feature vector.

Bhavneet Kaur et al. (2014) used the OPEN CV platform since it provides a C interface to implement various image processing algorithms. The work merges the feature extraction technique with this most suitable platform available for image algorithms.

\section{LOCAL BINARY PATTERN}

Local binary patterns (LBP) is a type of visual descriptor used for classification in computer vision. LBP is the particular case of the Texture Spectrum model proposed in 1990. LBP was first described in 1994. It has since been found to be a powerful feature for texture classification; it has further been determined that when LBP is combined with the Histogram of oriented gradients (HOG) descriptor, it improves the detection performance considerably on some datasets.

The Local Binary Patterns algorithm has its roots in 2D texture analysis. The basic idea is to summarize the local structure in an image by comparing each pixel with its neighborhood. Take a pixel as center and threshold its neighbors against. If the intensity of the center pixel is greater-equal its neighbor, then denote it with 1 and 0 if not. You'll end up with a binary number for each pixel, just like 11001111 . With 8 surrounding pixels you'll end up with $2^{\wedge} 8$ possible combinations, which are called Local Binary Patterns or sometimes abbreviated as LBP codes.

The first LBP operator actually used a fixed $3 \times 3$ neighborhood just like this: 


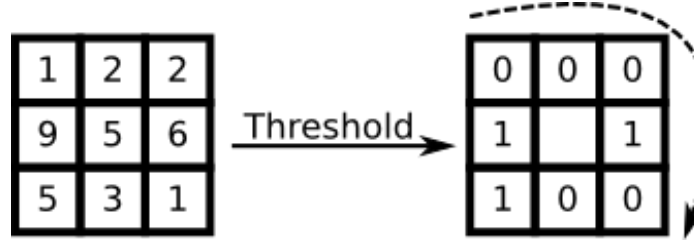

Binary: 00010011

Decimal: 19

Figure 3. Representation of Local Binary Pattern

The LBP feature vector, in its simplest form, is created in the following manner:

- $\quad$ Divide the examined window into cells (e.g. 16x16 pixels for each cell).

- For each pixel in a cell, compare the pixel to each of its 8 neighbors (on its left-top, left-middle, left-bottom, right-top, etc.). Follow the pixels along a circle, i.e. clockwise or counter-clockwise.

- Where the center pixel's value is greater than the neighbor's value, write "0". Otherwise, write "1". This gives an 8 digit binary number (which is usually converted to decimal for convenience).

- Compute the histogram, over the cell, of the frequency of each "number" occurring (i.e., each combination of which pixels are smaller and which are greater than the center). This histogram can be seen as a 256 -dimensional feature vector.

- Optionally normalize the histogram.

- Concatenate (normalized) histograms of all cells. This gives a feature vector for the entire window.

\section{RESEARCH MOTIVATION}

In many image processing applications, such as segmentation and classification, the selection of robust features descriptors is crucial to improve the discrimination capabilities in real world scenarios. Local Binary Patterns (LBPs) have been used in a wide range of texture classification scenarios and have proven to provide a highly discriminative feature representation.In particular, it is well known that image textures constitute power visual cues for feature extraction and classification. In the past few years the local binary pattern (LBP) approach, a texture descriptor method has gained increased acceptance due to its computational simplicity and more importantly for encoding a powerful signature for describing textures. However, the original algorithm presents some limitations such as low rate of precision and recall and its lack of rotational invariance which have led to many proposals or extensions in order to overcome such limitations. We will try to improve the precision and recall in the future work by using the classification mechanism like support vector machine.

\section{OPENCV}

The open source computer vision library, OpenCV, began as a research project at Intel in 1998. It has been available since 2000 under the BSD open source license. OpenCV is aimed at providing the tools needed to solve computer-vision problems. It contains a mix of low-level image-processing functions and high-level algorithms such as face detection, pedestrian detection, feature matching, and tracking. The library has been downloaded more than three million times. In 2010 a new module that provides GPU acceleration was added to OpenCV.

\section{CONCLUSION}

As we can see from the above descriptions, many visual features have been explored, both previously in computer vision applications and currently in image retrieval applications. For each visual feature, there exist multiple representations which model the human perception of that feature from different perspectives. What features and representations should be used in image retrieval is application dependent. There is a need of developing an image content description (model) to organize the features. It will specify a standard set of descriptors (feature representations) that can be used to describe various types of multimedia information. In soft query processing, the results reported are at the preliminary stages, in next stages investigation is required for alternative clustering and indexing techniques to reduce the complexity and storage requirements of our approach. Content based image retrieval by integration of metadata encoded multimedia features describes a new method for integrating multimedia text and image content features to increase the retrieval performance of the system. In Object based image retrieval, the approach works reasonably well. As such new approach must be required to handle millions of images in today's technological environment, but not hundreds of millions.

\section{REFERENCES}

[1] M. Fakheri, T. Sedghi, M. . G. Shayesteh1 and M. C. Amirani, "Framework for image retrieval using machine learning and statistical similarity matching techniques," IET Image Process, pp. 1-11, 2013.

[2] P. MANIPOONCHELVI and K. MUNEESWARAN, "Multi region based image retrieval system," Indian Academy of Sciences, pp. 333-344, 2014.

[3] H. J'egou, M. Douze, C. Schmid and P. P'erez, "Aggregating local descriptors into a compact image representation," 
IEEE, pp. 3304-3311, 2010.

[4] Y. Chen, J. . Z. Wang and R. Krovetz, "CLUE: Cluster-Based Retrieval of Images by Unsupervised Learning," IEEE, pp. 1187-1201, 2005.

[5] R. Fergus, L. Fei-Fei, P. Perona and A. Zisserman, "Learning Object Categories from Google's Image Search," IEEE, 2005.

[6] Y. Chen, X. Li, A. Dick and A. v. d. Hengel, "Boosting Object Retrieval With Group Queries," IEEE, pp. 765-768, 2012.

[7] R. Arandjelovi'c and A. Zisserman, "Three things everyone should know to improve object retrieval," IEEE, pp. 29112918, 2012.

[8] M. Perd'och, Chum and J. Matas, IEEE, pp. 9-16, 2009.

[9] S. . A. Chatzichristofis and Y. S. Boutalis, "CEDD: Color and Edge Directivity Descriptor: A Compact Descriptor for Image Indexing and Retrieval," Springer-Verlag Berlin Heidelberg, pp. 313-322, 2008.

[10] Y. Chen and J. Z. Wang, "A Region-Based Fuzzy Feature Matching Approach to Content-Based Image Retrieval," IEEE, pp. 1252-1267, 2002.

[11] C.-H. Lin, R.-T. Chen and Y.-K. Chan, "A smart content-based image retrieval system based on color and texture feature," ELSEVIER, p. 658-665, 2009.

[12] J. Z. Wang, J. Li and G. Wiederhold, "SIMPLIcity: Semantics-Sensitive Integrated Matching for Picture LIbraries," IEEE, pp. 947-963, 2001.

[13] S. Gandhani, R. Bhujade and A. Sinhal, "AN IMPROVED AND EFFICIENT IMPLEMENTATION OF CBIR SYSTEM BASED ON COMBINED FEATURES," IET, pp. 353-359.

[14] S. M. H. Khan, A. Hussain and I. F. T. Alshaikhl, "Comparative study on Content-Based Image Retrieval (CBIR)," IEEE, pp. 61-66, 2013.

[15] Sreedevi S. and Shinto Sebastian, "Fast Image Retrieval with Feature Levels," IEEE, 2013.

[16] S. Ezekiel, Mark G. Alford, David Ferris and Eric Jones,, "Multi-Scale Decomposition Tool for Content Based Image Retrieval," IEEE, 2013.

[17] K. Juneja, A. Verma, S. Goel and S. Goel , "A Survey on Recent Image Indexing and Retrieval Techniques for Lowlevel Feature Extraction in CBIR systems," IEEE, pp. 67-72, 2015.

[18] K. BELATTAR and S. MOSTEFAI, "CBIR using Relevance Feedback:Comparative Analysis and Major Challenges," IEEE, pp. 317-325, 2013.

[19] D. Jeyabharathi and A. Suruliandi, "Performance Analysis of Feature Extraction and Classification Techniques in CBIR," IEEE, pp. 1211-1214, 2013.

[20] H. Xie, Y. Ji and Y. Lu, "An Analogy-Relevance Feedback CBIR Method Using Multiple Features," IEEE, pp. 83-86, 2013.

[21] B. Kaur and S. Jindal, "An implementation of Feature Extraction over medical images on OPEN CV Environment".

[22] S. Kumar, S. Jain and T. Zaveri, "ARALLEL APPROACH TO EXPEDITE MORPHOLOGICAL FEATURE EXTRACTION OF REMOTE SENSING IMAGES FOR CBIR SYSTEM," IEEE, pp. 2471-2474, 2014.

[23] K. BELATTAR and S. MOSTEFAI , "CBIR with RF:which Technique for which Image," IEEE, 2013.

[24] G. Raghuwanshi and V. Tyagi, "Texture image retrieval using adaptive tetrolet transforms," ELSEVIER, pp. 1-8, 2015. 